\title{
The assesment of prothrombotic potential using thrombin generation assay in pediatric patients with nephrotic syndrome: preliminary study
}

\author{
Eren Soyaltın ${ }^{1 \oplus}, Y_{1} 1 \mathrm{maz} \mathrm{Ay}^{2 \oplus}$, Erkin Serdaroğlu' ${ }^{1 \oplus}$, Raziye Canan Vergin ${ }^{2 \oplus}$ \\ Divisions of ${ }^{1}$ Nephrology and ${ }^{2}$ Hematology, Department of Pediatrics, University of Health Sciences, Dr. Behcet Uz Child Disease and \\ Pediatric Surgery Training and Research Hospital, Izmir, Turkey.
}

\begin{abstract}
Background. Nephrotic syndrome (NS) is a common kidney disease associated with an increased risk of thrombotic events. The aim of this study was to assess the prothrombotic potential of patients with NS using the thrombin generation assay (TGA).

Methods. A total of 35 patients with NS, who were followed in the Division of Pediatric Nephrology in Behcet Uz Children's Hospital, were included in the study. After the patients with Steroid Resistant NS (n:3) were excluded, 32 patients in total were evaluated for TGA. Patients were primarily classified according to their response to corticosteroid therapy. The control group consisted of 34 healthy volunteers with similar gender and age distribution to the patients. Blood urine nitrogen, creatinine, albumin, triglyceride, cholesterol, 24-hour proteinuria, platelets, erythrocyte sedimentation rate, C-reactive protein and thrombin generation values in activation and remission period of NS were compared. Moreover, TGA values of the patients in their remission period were compared with the values of those in the control group.
\end{abstract}

Results. Endogenous thrombin potential (ETP) and peak thrombin levels were significantly higher in the activation period than remission period of NS. Additionally, after the patients achieved remission, their ETP was still higher than the control group. There was a negative correlation between both ETP and peak thrombin levels of patients with serum albumin, whereas a significant positive correlation was detected with platelet levels. Thromboembolic events were not observed in any of the patients during follow-up.

Conclusions. Nephrotic syndrome is strongly associated with hypercoagulopathy as assessed by TGA during active NS. The present study reinforces the usefulness of TGA as a marker of hypercoagulability in pediatric patients with NS. Further studies are needed in this regard.

Key words: nephrotic syndrome, thrombin generation assay, children, hypercoagulability.

Nephrotic syndrome (NS) is a disorder of the kidneys which originates from increased permeability of the glomerular filtration barrier. It is characterized by proteinuria, hypoalbuminemia, edema and hyperlipidemia. ${ }^{1}$ NS is associated with hypercoagulability and an elevated risk of thromboembolic events

\footnotetext{
Eren Soyaltın

erensoyaltin@hotmail.com
}

Received 16th April 2020, revised 30th May 2020, 8th July 2020, 19th August 2020, 23rd September 2020, accepted 7th October 2020. leading to increased morbidity and mortality. ${ }^{2}$ The thromboembolic event incidence in NS has been reported as $3-44 \%$ depending on the extent of the diagnostic screening. ${ }^{3}$ Pulmonary embolism and renal vein thrombosis have been reported as common complications (20-35\%) in patients with NS.4-6 The incidence of subclinical pulmonary embolism by scintigraphic pulmonary ventilation and perfusion studies was found to be $28 \%$ in children with NS. ${ }^{7}$ Additionally, most patients (adults and children) with pulmonary embolism (84\%) were asymptomatic. ${ }^{8-11}$ 
The risk of thrombosis in patients diagnosed with NS can be affected by multiple factors and can be divided into two categories; urinary loss of proteins and increased hepatic synthesis of procoagulant proteins. ${ }^{12,13}$ The urinary loss of proteins, such as antithrombin, plasminogen, protein $C$ and $S$ and increased synthesis of factors I, II, V, VII, VIII, X, and XIII promote thrombosis. ${ }^{13}$ Thrombocytosis and increased platelet aggregation in NS can also play a role in thrombosis. ${ }^{14}$ Furthermore, hyperviscosity and hyperlipidemia can also be responsible for the prothrombotic state. ${ }^{15}$

Treatment of NS such as corticosteroids, diuretics and usage of a central venous catheter, can also result in an increased risk of venous thromboembolism (VTE). Many studies have demonstrated the relationship between the hemostatic status of patients in activation and remission period of NS with healthy individuals. ${ }^{13-17}$ However, no study has yet been reported on the thrombin generation assay (TGA) which indicates global hemostasis and the result of these changes during NS in children.

Thrombin is a multifunctional protein and a key enzyme in the coagulation system. It is involved in coagulation, anticoagulation, endothelial activation and proliferation of fibroblasts. With the use of TGA, the endogenous capacity of the overall hemostatic potential can be evaluated. Both thrombotic conditions due to high thrombin production and the hemorrhagic conditions due to low thrombin production can be assessed. It is possible to evaluate the relationship between both proteases and inhibitors by measuring the thrombin capacity with TGA. ${ }^{18,19}$

In this study, we aimed to evaluate the prothrombotic potential increase in the activation and remission periods of patients with NS with TGA, a test that measures hypercoagulation by measuring thrombin formation capacity.

\section{Material and Methods}

The study was carried out from March 2015 to August 2015 and included 35 patients in total, who were followed with NS in the Division of Pediatric Nephrology in Behcet Uz Children's Hospital. This retrospective analysis was approved by the Institutional Review Board and approval was received from the local Ethics Committee (2015/04-04). The study was approved on 12.03.2015. Following approval from the ethics committee; patients, volunteers and their families were informed, and written consent was received.

Patients who were admitted to hospital during the study period in activation of idiopathic NS were included and the following data was collected: age, gender, response to therapy, duration of follow-up, additional diseases, and history of personal and/or familial thromboembolic events. The activation period was described as nephrotic range of proteinuria, hypoalbuminemia and edema; remission of the disease was described as urinary protein analysis in 24 hours $<4 \mathrm{mg} / \mathrm{m}^{2} /$ hour or urine protein to creatinine ratio $<0.2 \mathrm{mg} / \mathrm{mg}$ creatinine for 3 consecutive days, and normal values of serum albumin. Patients were primarily classified by their response to corticosteroid therapy as; corticosteroid responsive (steroid sensitive): if complete remission with corticosteroid therapy was attained; or steroid resistant (SR): if there was inability to induce a remission within 4-6 weeks of daily corticosteroid therapy ( $2 \mathrm{mg}$ / $\mathrm{kg} /$ day or $60 \mathrm{mg} / \mathrm{m}^{2} /$ day). Steroid responsive patients who relapse during the dose tapering or within 2 weeks of discontinuation of steroid therapy are termed steroid dependent NS. Patients diagnosed with SRNS (n:3) were excluded and 32 patients were evaluated for TGA in the study (Fig 1). None of the patients or their parents had a history of thrombosis. During the study period, the anticoagulant prophylaxis was not used in the patients.

Urea nitrogen, creatinine, albumin, triglyceride, cholesterol, 24-hour quantitative proteinuria, platelets, erythrocyte sedimentation rate, 


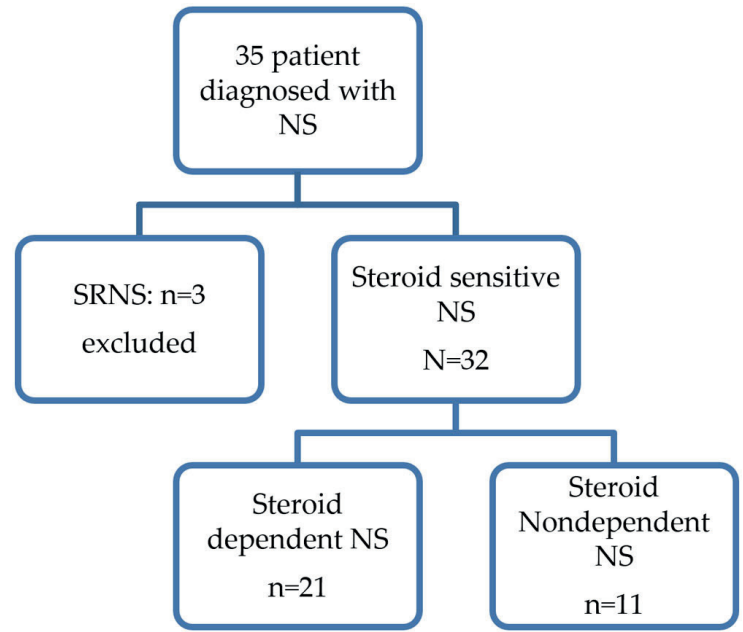

Fig. 1. Diagnoses of patients in study. NS: nephrotic syndrome

C-reactive protein and TG were assessed during hospital admission with activation of NS.

After the patients achieved remission, TG was assessed again for comparison. The patients' and healthy volunteers' blood pressures were measured during their visits. The control group consisted of 34 healthy children with no glomerulopathy, acute infection or family history of thrombosis. They were admitted to the pediatric nephrology clinic for urological pathologies (monosymptomatic enuresis or isolated hydronephrosis) with normal renal function and biochemical parameters and did not use any medication.

Blood sampling procedure and organizing Platelet-Poor Plasma (PPP): Peripheral venous blood was collected into vacutainer tubes containing $0.129 \mathrm{~mol} / \mathrm{L}$ tri-sodium citrate. With a double centrifugation at $2500 \mathrm{~g}$ for 15 minutes, PPP was collected from the upper half of the plasma supernatant, frozen and stored at $-80{ }^{\circ} \mathrm{C}$. For the TGA test, aliquoted PPP was stored for 3 months at $-80{ }^{\circ} \mathrm{C}$ and all samples were assessed simultaneously. The Calibrated Automated Thrombogram method was used for TGA. ${ }^{14}$ Recombinant relipidated tissue factor Innovin was used to activate thrombin generation. The assays were performed according to the manufacturers' instructions for thrombin generation with a mixture of $5 \mathrm{pM}$ TF and $4 \mu \mathrm{M}$ phospholipids for PPP. Fluorescence was measured using an Ascent Reader, and thrombin generation curves were calculated with the Thrombinoscope ${ }^{\mathrm{TM}}$ software package. Thrombin generation parameters include the following: lag time (time to $16.7 \%$ of peak concentration; $\mathrm{min}$ ), endogenous thrombin potential(ETP; area under the curve; nMminute), peak thrombin (nM), time to peak (min), and start to tail (min). ${ }^{20}$ The 24-hour urine samples which were collected from the patients were used to determine protein excretion. Thrombin generation parameters of patients during the active NS period were compared with the parameters during the remission period. The interval between activation and remission was a minimum of four weeks. Thrombin generation parameters in remission period of the patients were compared with the thrombin generation parameters of the healthy volunteers, and it was evaluated whether the patients were susceptible to hypercoagulability even in the remission period. Patients with steroid-dependent NS and non-dependent NS were also compared among each other.

Statistical analyses were carried out using SPSS 18.0. Normality of the variable distribution was evaluated using parametric tests. Variables were analyzed by means of nonparametric tests if not normally distributed. Measurements normally distributed were reported as the mean $\pm \mathrm{SD}$; non-normally distributed data were expressed as median and minimal-maximal value. The Student's $t$ test or Mann-Whitney U test were used in statistical analysis to compare differences between groups. Pearson correlation analysis was used for correlation of the parameters in active disease and TGA values. $P$ values $<0.05$ were considered statistically significant.

\section{Results}

The study group consisted of 32 patients with NS (18 male, 14 female). The mean age of the participants was $128 \pm 53$ months. There was no history of thromboembolic events in any 
participants or their parents. The control group was comprised of 16 males and 18 females. Similarly, the mean age was $125 \pm 53$ months (Table I). The laboratory results of patients with NS in activation and remission period are presented in Table II.

From the evaluation of TGA parameters in activation and remission periods of patients showed that; ETP and peak thrombin levels were significantly higher during the activation period compared to remission period ( $\mathrm{P}<0.001$, and $\mathrm{P}<0.001$, respectively) (Table III). Although patients achieved remission, their ETP levels were still higher than the control group ( $P$ $=0.001)$. Mean lag time of the patients in activation period was significantly shorter than in remission $(p<0.001)$. Additionally, mean time to peak and mean start tail levels of the patients were shorter in remission compared to activation ( $\mathrm{P}<0.001$ and $\mathrm{P}<0.001$, respectively) (Table III). Conversely, lag time and time to peak of the patients with remission were longer than the control group $(\mathrm{P}=0.001$ and $\mathrm{P}=0.017$, respectively) (Table III). In a comparison of the TGA parameters of patients diagnosed with the subgroups of steroid sensitive NS, there was no significant difference between steroid dependent and non-steroid dependent disease groups (Table IV).

A significant converse correlation was found between serum albumin and both ETP and peak thrombin of the patients $(\mathrm{P}<0.001$ and $\mathrm{P}=0.005$, respectively), while there was a positive correlation between serum albumin and start tail $(\mathrm{P}=0.010)$. In addition, there was a negative relationship between urine protein of the patients and lag time, time to peak and start tail, while a significant positive correlation was

Table I. The demographic and clinical data of patients with NS and healthy volunteers.

\begin{tabular}{lccc}
\hline & NS patients (n: 32) & Healthy volunteers (n: 34) & $\mathrm{p}$ \\
\hline Age (month) & $128 \pm 53$ & $125 \pm 54$ & 0.78 \\
Gender & & & 0.40 \\
$\quad$ Female & 14 & 18 & - \\
$\quad$ Male & 18 & - & \\
Other chronic disease & - & - & \\
Tromboembolic event & - & - & \\
Tromboembolic event in family members & &
\end{tabular}

NS: nephrotic syndrome

Table II. The laboratory data of patients with NS and healthy volunteers.

\begin{tabular}{lcc}
\hline \multirow{2}{*}{ Laboratory parameters } & \multicolumn{2}{c}{ NS patients (n: 32) } \\
\cline { 2 - 3 } & Active disease period & Remission period \\
\hline BUN $(\mathrm{mg} / \mathrm{dl})$ & $13.0 \pm 7.4$ & $9.6 \pm 2.6$ \\
Creatinine $(\mathrm{mg} / \mathrm{dl})$ & $0.51 \pm 0.12$ & $0.50 \pm 0.10$ \\
Albumine $(\mathrm{g} / \mathrm{dl})$ & $2.5 \pm 0.7$ & $4.0 \pm 0.39$ \\
Total cholesterole (mg/dl) & $331.3 \pm 107.3$ & $207.5 \pm 70.4$ \\
LDL (mg/dl) & $206 \pm 8.0$ & $128 \pm 56$ \\
HDL $(\mathrm{mg} / \mathrm{dl})$ & $63 \pm 16$ & $69 \pm 17.8$ \\
Triglycerides $(\mathrm{mg} / \mathrm{dl})$ & $243 \pm 145$ & $207 \pm 70$ \\
Platelets $\left(/ \mathrm{mm}{ }^{3}\right)$ & $390.228 \pm 100$ & $337.571 \pm 76.050$ \\
Sedimentation $(\mathrm{mm} / \mathrm{hour})$ & $47.2 \pm 1.9$ & $20.7 \pm 13.6$ \\
24 hour urine protein $\left(\mathrm{mg} / \mathrm{m}^{2} /\right.$ hour $)$ & $140 \pm 77$ & $2.2 \pm 1.4$
\end{tabular}

NS: nephrotic syndrome, mg: milligram, dl: deciliter, m: meter 
Table III. Comparison of TGA parameters in patients with NS during activation, remission and healthy control group.

\begin{tabular}{|c|c|c|c|c|c|}
\hline & $\begin{array}{c}\text { Activation of NS } \\
\text { Mean } \pm \text { SD }\end{array}$ & $\begin{array}{c}\text { Remission of NS } \\
\text { Mean } \pm \text { SD }\end{array}$ & $\begin{array}{c}\text { Control } \\
\text { Mean } \pm \text { SD }\end{array}$ & $\begin{array}{c}\mathrm{p} 1 \\
\mathrm{~A} / \mathrm{R}\end{array}$ & $\begin{array}{l}\mathrm{p} 2 \\
\mathrm{R} / \mathrm{C}\end{array}$ \\
\hline Lag time (min) & $4.4 \pm 1.2$ & $5.9 \pm 2$ & $4.5 \pm 1.1$ & $<0.001$ & 0.001 \\
\hline $\operatorname{ETP}(\mathrm{nM} / \mathrm{dk})$ & $2550 \pm 494$ & $1715 \pm 467$ & $1355 \pm 386$ & $<0.001$ & 0.001 \\
\hline Peak thrombin (nM) & $356 \pm 96$ & $209 \pm 91$ & $197 \pm 80$ & $<0.001$ & 0.575 \\
\hline Time to peak (min) & $8.9 \pm 1.7$ & $11.2 \pm 3.0$ & $9.3 \pm 3.2$ & $<0.001$ & 0.017 \\
\hline Start to tail (min) & $20.2 \pm 3.0$ & $28.2 \pm 8.9$ & $24.3 \pm 8.5$ & $<0.001$ & 0.073 \\
\hline
\end{tabular}

TGA: thrombin generation assay, ETP: endogenous thrombin potential, NS: nephrotic syndrome, A: activation, R: remission, C: control, SD: standard deviation, min: minute

( $\mathrm{p} 1$ value for: statistical significance between TGA parameters of patients during activation and remission periods,

p2 value for: statistical significance between patients' TGA values during remission and healthy children's TGA values.)

Table IV. Comparison of TGA parameters in patients with steroid dependent NS and steroid responsive (not dependent) NS patients during active disease period.

\begin{tabular}{lccc}
\hline & $\begin{array}{c}\text { Steroid dependent NS } \\
\text { patients } \\
\text { Mean } \pm \text { SD (n: 21) }\end{array}$ & $\begin{array}{c}\text { Steroid not dependent } \\
\text { NS patients } \\
\text { Mean } \pm \text { SD (n: 11) }\end{array}$ & p \\
\hline Age & $10.36 \pm 3.5$ & $11.52 \pm 5.0$ & 0.502 \\
Gender & & & \\
$\quad$ Female/Male & $13 / 8$ & $1 / 10$ & 0.297 \\
Follow up with NS period (year) & $7.02 \pm 5.4$ & $5.13 \pm 2.9$ & \\
Other chronic disease & - & - & \\
Tromboembolic event & - & - & 0.598 \\
Tromboembolic event in family members & - & $4.1 \pm 0.98$ & 0.646 \\
Lag time (min) & $4.3 \pm 1.1$ & $2510 \pm 348$ & 0.302 \\
ETP (nM/dk) & $2599 \pm 579$ & $380 \pm 55.5$ & 0.963 \\
Peak thrombin (nM) & $348 \pm 116$ & $8.3 \pm 0.85$ & 0.350 \\
Time to peak (min) & $8.9 \pm 1.9$ & $20.5 \pm 3.5$ & \\
Start to tail (min) & $20.5 \pm 3.5$ & & \\
\hline
\end{tabular}

TGA: thrombin generation assay, ETP: endogenous thrombin potential, NS: nephrotic syndrome, SD: standard deviation, min: minute

detected between proteinuria and both ETP and peak thrombin (Table V). A significant positive correlation was found between triglyceride of the patients and both ETP and peak thrombin $(\mathrm{P}=0.003$, and $\mathrm{P}=0.006$, respectively) (Table $\mathrm{V})$.

A significant relationship between peak thrombin and cholesterol was also observed $(P=0.007)$, whereas no correlation between cholesterol and ETP was found $(\mathrm{P}=0.428)$.

Thrombocytosis is also defined as a platelet count greater than two standard deviations above the mean or greater than $450,000 / \mathrm{mm}^{3} .{ }^{21}$ According to this definition, there were no patients with thrombocytosis in our study; but platelet counts of the patients with NS in activation $(390.228 \pm$ $100 / \mathrm{mm}^{3}$ ) were significantly higher than the control group $\left(337.571 \pm 76 / \mathrm{mm}^{3}\right) \quad(\mathrm{p}<0,001)$. There was a significant positive correlation between platelet counts of the patients and both ETP and peak thrombin $(\mathrm{P}=0.001$ and $\mathrm{P}=0.006$, respectively) (Table V).

Thromboembolic event was not observed in any of the patients during follow-up. 
Table V. The correlation between serum total cholesterol, triglyceride, platelets of the patients and TGA parameters.

\begin{tabular}{lccccccccccc}
\hline & \multicolumn{2}{c}{ T. cholesterol } & \multicolumn{2}{c}{ Triglyceride } & \multicolumn{2}{c}{ S. albumine } & \multicolumn{2}{c}{ U. protein } & \multicolumn{2}{c}{ Platelets } \\
\cline { 2 - 13 } & $\mathrm{R}$ & $\mathrm{P}$ & $\mathrm{R}$ & $\mathrm{P}$ & $\mathrm{R}$ & $\mathrm{P}$ & $\mathrm{R}$ & $\mathrm{P}$ & $\mathrm{R}$ & $\mathrm{P}$ \\
\hline Lag time(min) & -0.143 & 0.238 & -0.134 & 0.270 & 0.158 & 0.191 & -0.239 & 0.470 & -0.340 & 0.778 \\
ETP (nM/dk) & 0.000 & 0.428 & 0.346 & 0.003 & -0.477 & $<0.001$ & 0.561 & $<0.001$ & 0.404 & 0.001 \\
PT (nM) & 0.322 & 0.007 & 0.325 & 0.006 & -0.331 & 0.005 & 0.561 & $<0.001$ & 0.327 & 0.006 \\
Time to peak (min) & -0.099 & 0.415 & -0.175 & 0.147 & 0.132 & 0.277 & 0.298 & 0.120 & -0.138 & 0.254 \\
Start to tail (min) & -0.288 & 0.016 & -0.230 & 0.056 & 0.307 & 0.010 & -0.404 & 0.001 & -0.162 & 0.180
\end{tabular}

TGA: thrombin generation assay, ETP: endogenous thrombin potential, PT: peak thrombin, T.cholesterol: total cholesterol, S. albumine: serum albumin, U. protein: urine protein

( $\mathrm{R}<0.2$ : too weak correlation, 0.2-0.4: Poor correlation, 0.4-0.6: Moderate correlation, 0.6-0.8: High correlation, :0.8> Very high correlation)

\section{Discussion}

NS is a common kidney disease associated with a significantly increased risk of thrombotic events. The important changes in plasma hemostatic proteins (such as increased factor V, factor VIII, fibrinogen, factor XI, $\alpha 2$ macroglobulin, decreased protein $C$, protein $\mathrm{S}$ and antithrombin) in patients with NS have been shown in several studies. ${ }^{17-19,22}$ Although hemostatic potential is increased in NS, there are very few studies about direct examination of global hemostasis.

Kerlin et al. ${ }^{12}$ reported that thrombotic capacity is correlated with disease severity, but evident thrombosis may necessitate vascular injury (twohit hypothesis). In the same study, they have shown that TGA has a significant correlation with the severity of hypoalbuminemia and proteinuria. $^{13}$ Their results suggest that the increased thrombin-generating capacity is responsible for susceptibility to thrombotic events and therefore, the risk of thrombosis increases gradually depending on the severity of the disease. Similar to the report of Kerlin et al. ${ }^{12}$; urinary protein excretion, serum albumin and triglyceride levels correlated significantly with ETP and peak thrombin levels of the patients in our study. ETP and peak thrombin levels of the patients in activation were significantly higher than those in remission. Mean lag time, time to peak and start tail of the patients in activation was significantly shorter than in remission.
Additionally, although the patients achieved remission, mean ETP levels were still higher than the control group. In recent studies, it has been reported that the increase in anticoagulant factors and decrease in procoagulant factors return to normal levels after remission. ${ }^{22-24}$ However, the persistence of hypercoagulability after remission and the changes in factor levels have not been reported.

Despite a significant high thrombotic capacity in patients with NS in activation, thromboembolic event was not observed during follow-up. These results may be correlative with a two-hit hypothesis of thrombus expansion, which has been offered for other prothrombotic disorders (e.g., antiphospholipid syndrome). An acquired prothrombotic coagulopathy (first hit) is not adequate to induce thrombosis in the absence of a second event, such as vascular injury (second hit). ${ }^{25}$ Consequently, more studies should examine the multifactorial effects of both disease severity and other prothrombotic conditions such as cardiovascular disease, catheter-related venous endothelial injury, trauma or venous stasis related to edema.

Increased thrombin has been shown in a single human study (adults) of NS. ${ }^{26}$ Mahmoodi et al. ${ }^{26}$ reported that peak thrombin, in the presence of thrombomodulin, was significantly lower during the antiproteinuric treatment period compared to the placebo. Additionally, ETP, peak thrombin and time to peak demonstrated 
a more procoagulant state in patients with NS than controls. However, mean levels of lag time and time to peak were similar during the antiproteinuric treatment and the placebo period. ${ }^{26}$ Similarly, in thisstudy, TGA parameters in NS with activation were significantly higher than both in remission and control groups. Although the patients achieved remission, their ETP was still higher than the healthy control group. These findings may indicate that even if remission is obtained in NS, the prothrombotic state goes on. Conversely, lag time and time to peak of the patients in remission were longer than the healthy control group. Because of this discrepancy in the findings about lag time and peak thrombin, these TGA parameters may not be useful to demonstrate nephrotic hypercoagulopathy.

When the TGA parameters in activation of corticosteroiddependent, corticosteroid resistant and relapse groups were compared with each other, there was no significant difference. These results may show that activation of the disease is the basis of hypercoagulability condition in NS rather than response to corticosteroid. Both severity of hypoalbuminemia and proteinuria have been accompanying factors to thrombotic risk. ${ }^{27}$ In another pediatric study, worsening proteinuria is directly correlated with increasing venous thrombosis probability. ${ }^{3}$ However, hypoalbuminemia, which is closely correlated to proteinuria severity, was reported not to be a significant marker in a recent large pediatric cohort. ${ }^{3}$ We revealed that ETP and peak thrombin significantly correlated with serum albumin and proteinuria of the patients. Although TGA may be a marker for thrombotic risk, its clinical practice has been limited due to lack of standardization among test techniques. ${ }^{26,28}$ Thus, proteinuria and/or serum albumin may become suitable and useful biomarkers for identifying those patients with an increased risk of thrombosis. ${ }^{27,29}$

Hyperlipidemia is a known factor underlying atherosclerosis. Nevertheless, it is unclear whether it is a cause of thrombosis or not. Paraskevas et al. ${ }^{30}$ revealed that statins are able to lower the risk of venous thrombosis in NS patients when compared with a placebo. ${ }^{30}$ These actions of statins have been explained with their anti-inflammatory effects, prevention of atherosclerotic vascular diseases and modulation of the coagulation cascade. ${ }^{31}$ In this study, we showed that there was a significant positive correlation between triglyceride of the patients and both ETP and peak thrombin. Total cholesterol also had a significant relationship with peak thrombin, but there was no correlation with ETP. These results indicate that hyperlipidemia in activation period of NS may increase thrombin generation, which is the reason for the hypercoagulable condition. Increased platelet counts and hyperactivity have been detected in children with NS. ${ }^{30,32}$ Although these findings may show increased risk for thrombotic event especially in adults with atherosclerotic disease, reactive thrombocytosis is rarely a cause for thrombotic event in children. ${ }^{33-35}$

The underlying linkage between platelet alterations and the occurrence of thromboembolic event is not completely understood. However, Eneman et al. ${ }^{14}$ have shown that the mechanisms underlying platelet abnormalities are probably due to changes in plasma levels of platelet-interfering proteins and lipids, as a result of NS. ${ }^{11}$ The pathogenesis of platelet hyperactivity is also associated with hypoalbuminemia, hyperfibrinogenemia, high levels of cholesterol and low-density lipoprotein. ${ }^{36-38}$ In this study, even if platelet function of the patients was not assessed, their platelet counts in activation were significantly higher than the control group. There was also a significant positive correlation between platelets of the patients in activation and both ETP and peak thrombin. Since platelet-rich plasma was not used for TGA, this correlation was thought to be related to the increase in platelet count as well as the increase in ETP and peak thrombin levels at the time of activation.

This study has several limitations. First, the number of patients was low. Second, the other inherited thrombosis risk factors that may be 
responsible for the prothrombotic state in NS (such as fibrinogen, FV, FVIII, FXI, AT, PS, PC, $\alpha 2$ macroglobulin, and plasminogen levels) were not assessed. Third, if TGA was also performed with platelet rich plasma, we could attain more information regarding nephrotic hypercoagulopathy.

In conclusion, this is the first pediatric study that shows prothrombotic state by TGA in NS. This study will help to identify children with NS who are at high risk of thrombosis. We think that the patients who have other prothrombotic circumstances (such as trauma, obesity, cardiovascular disease, venous catheterrelated endothelial injury, or bed rest) should be closely monitored for thrombotic events. TGA seems a promising laboratory tool for investigating patients with risk of thrombosis or VTE recurrence but further studies with standardized TGA are needed to make it applicable in clinical practice.

\section{REFERENCES}

1. Andolino TP, Reid-Adam J. Nephrotic syndrome. Pediatr Rev 2015; 36: 117-125.

2. Kelddal S, Nykjær KM, Gregersen JW, Birn H. Prophylactic anticoagulation in nephrotic syndrome prevents thromboembolic complications. BMC Nephrol 2019; 20: 139.

3. Rankin AJ, McQuarrie EP, Fox JG, Geddes CC, MacKinnon B; Scottish Renal Biopsy Registry. Venous thromboembolism in primary nephrotic syndrome - is the risk high enough to justify prophylactic anticoagulation? Nephron 2017; 135: $39-45$.

4. Zhang LJ, Zhang Z, Li SJ, et al. Pulmonary embolism and renal vein thrombosis in patients with nephrotic syndrome: prospective evaluation of prevalence and risk factors with CT. Radiology 2014; 273: 897-906.

5. Eddy AA, Symons JM. Nephrotic syndrome in childhood. Lancet2003; 362: 629-639.

6. Orth SR, Ritz E. The nephrotic syndrome. N Engl J Med 1998; 338: 1202-1211.

7. Cherng SC, Huang WS, Wang YF, Yang SP, Lin YF. The role of lung scintigraphy in the diagnosis of nephrotic syndrome with pulmonary embolism. Clin Nucl Med 2000; 25: 167-172.
8. Mahan JD, Mauer SM, Sibley RK, Vernier RL. Congenital nephrotic syndrome: evolution of medical management and results of renal transplantation. J Pediatr 1984; 105: 549-557.

9. Kerlin BA, Blatt NB, Fuh B, et al. Epidemiology and risk factors for thromboembolic complications of childhood nephrotic syndrome: a Midwest Pediatric Nephrology Consortium (MWPNC) study. J Pediatr 2009; 155: 105-110.

10. Kerlin BA, Ayoob R, Smoyer WE. Epidemiology and pathophysiology of nephrotic syndrome-associated thromboembolic disease. Clin J Am Soc Nephrol 2012; 7: 513-520.

11. Zhang LJ, Zhang Z, Li SJ, et al. Pulmonary embolism and renal vein thrombosis in patients with nephrotic syndrome: prospective evaluation of prevalence and risk factors with CT. Radiology 2014; 273: 897-906.

12. Kerlin BA, Haworth K, Smoyer WE. Venous thromboembolism in pediatric nephrotic syndrome. Pediatr Nephrol 2014; 29: 989-997.

13. Loscalzo J. Venous thrombosis in the nephrotic syndrome. N Engl J Med 2013; 368: 956-958.

14. Eneman B, Levtchenko E, van den Heuvel B, Van Geet C, Freson K. Platelet abnormalities in nephrotic syndrome. Pediatr Nephrol 2016; 31: 1267-1279.

15. Wang CS, Greenbaum LA. Nephrotic syndrome. Pediatr Clin North Am 2019; 66: 73-85

16. Kerlin BA, Waller AP, Sharma R, Chanley MA, Nieman MT, Smoyer WE. Disease severity correlates with thrombotic capacity in experimental nephrotic syndrome. J Am Soc Nephrol 2015; 26: 3009-3019.

17. Fabri D, Belangero VM, Annichino-Bizzacchi JM, Arruda VR. Inherited risk factors for thrombophilia in children with nephrotic syndrome. Eur J Pediatr 1998; 157: 939-942.

18. Chugh KS, Malik N, Uberoi HS, et al. Renal vein thrombosis in nephrotic syndrome-a prospective study and review. Postgrad Med J 1981; 57: 566-570.

19. Sá H, Freitas L, Mota A, Cunha F, Marques A Primary antiphospholipid syndrome presented by total infarction of right kidney with nephrotic syndrome. Clin Nephrol 1999; 52: 56-60.

20. Duarte RCF, Ferreira CN, Rios DRA, Reis HJ, Carvalhoa MDG. Thrombin generation assays for global evaluation of the hemostatic system: perspectives and limitations. Rev Bras Hematol Hemoter 2017; 39: 259-265.

21. Tefferi A, Vannucchi AM, Barbui T. Essential thrombocythemia treatment algorithm. Blood Cancer J 2018; 8: 2. 
22. Hemker HC, Giesen P, Al Dieri R, et al. Calibrated automated thrombin generation meausurement in clotting plasma. Pathophysiol Haemost Thromb 2003; 33: 4-15.

23. Gerotziafas GT, Depasse F, Busson J, Leflem L, Elalamy I, Samama MM. Towards a standardization of thrombin generation assessment: the influence of tissue factor, platelets and phospholipids concentration on the normal values of ThrombogramThrombinoscope assay. Thromb J 2005; 3: 16.

24. Schlegel N. Thromboembolic risks and complications in nephrotic children. Semin Thromb Hemost 1997; 23: $271-280$.

25. Citak A, Emre S, Sairin A, Bilge I, Nayir A. Hemostatic problems and thromboembolic complications in nephrotic children. Pediatr Nephrol 2000; 14: 138142.

26. Mahmoodi BK, Mulder AB, Waanders F, et al. The impact of antiproteinuric therapy on the prothrombotic state in patients with overt proteinuria. J Thromb Haemost 2011; 9: 2416-2423.

27. Ozkayin N, Mir S, Kavakli K. Hypercoagulability risk factors in children with minimal change disease and the protective role of protein-C activity. Int Urol Nephrol 2004; 36: 599-603.

28. Broder A, Tobin JN, Putterman C. High antiphospholipid antibody levels are associated with statin use and may reflect chronic endothelial damage in non-autoimmune thrombosis: crosssectional study. J Clin Pathol 2012; 65: 551-556.

29. Ozkaya O, Bek K, Fisgin T, et al. Low protein Z levels in children with nephrotic syndrome. Pediatr Nephrol 2006; 21: 1122-1126.

30. Paraskevas KI, Bessias N, Perdikides TP, Mikhailidis DP. Statins and venous thromboembolism: a novel effect of statins? Curr Med Res Opin 2009; 25: 18071809.
31. Dargaud Y, Luddington R, Gray E, et al Standardisation of thrombin generation testwhich reference plasma for TGT? An international multicentre study. Thromb Res 2010; 125: 353-356.

32. Dargaud Y, Wolberg AS, Luddington R, et al. Evaluation of a standardized protocol for thrombin generation measurement using the calibrated automated thrombogram: an international multicentre study. Thromb Res 2012; 130: 929-934.

33. Kumar S, Chapagain A, Nitsch D, Yaqoob MM Proteinuria and hypoalbuminemia are risk factors for thromboembolic events in patients with idiopathic membranous nephropathy: an observational study. BMC Nephrol 2012; 13: 107.

34. Resh M, Mahmoodi BK, Navis GJ, Veeger NJ, Lijfering WM. Statin use in patients with nephrotic syndrome is associated with a lower risk of venous thromboembolism. Thromb Res 2011; 127: 395-399.

35. Eddy AA, Symons JM. Nephrotic syndrome in childhood. Lancet 2003; 362: 629-639.

36. Walter E, Deppermann D, Andrassy K, Koderisch J. Platelet hyperaggregability as a consequence of the nephrotic syndrome. Thromb Res 1981; 23: 473-479.

37. Mahmoodi BK, ten Kate MK, Waanders F, et al. High absolute risks and predictors of venous and arterial thromboembolic events in patients with nephrotic syndrome: results from a large retrospective cohort study. Circulation 2008; 117: 224-230.

38. Dame C, Sutor AH. Primary and secondary thrombocytosis in childhood. Br J Haematol 2005; 129: 165-177. 